\title{
Percepção de brasileiros sobre morte digna
}

\section{Perception of Brazilians on death with dignity}

Artigo Original | Original Article

Cynthia de Freitas Melo, PhD (1a), Graciele Alves Moura Bezerra, PsyM (1b), Kelly Gomes Lima, PsyM (1b)

(1) Universidade de Fortaleza, Fortaleza/CE, Brasil

(a) Concepção do projeto, análise de dados e revisão do manuscrito.

(b) Escrita da parte teórica, coleta de dados, análise e discussão dos dados.

Nota. Artigo escrito em português do Brasil.

Autor para correspondência | Corresponding author: Cynthia Melo; cf.melo@yahoo.com.br

\section{RESUMO}

Palavras-Chave

Atitude frente à morte

Cuidados paliativos

Morte

Keywords

Attitude facing death

Palliative care

Death
Objetivos: A presente pesquisa objetivou averiguar a conceção de morte digna para a população brasileira.

Método: Foi realizada uma pesquisa exploratória, de levantamento nacional, de cunho quantitativo. Contou-se com uma amostra não probabilística acidental por conveniência composta por 412 brasileiros que responderam a "Escala de perceção de morte digna", cujos dados foram analisados por meio de estatística descritiva e bivariada com auxílio do pacote estatístico SPSS (Statistical Package for Social Science) for Windows versão 22.

Resultados: Foi possível verificar que a morte digna implica, por ordem de prioridade, uma boa relação com a família $(M$ $=5,99 ; D P=0,73)$, manutenção da esperança e do prazer $(M=5,88 ; D P=0,85)$, boa relação com a equipe profissional de saúde $(M=5,46 ; D P=1,00)$, não ser um fardo para os demais $(M=5,38 ; D P=1,09)$, ter controlo físico e cognitivo $(M$ $=4,69 ; D P=0,92)$ e ter controle do futuro $(M=4,57 ; D P=1,14)$.

Conclusões: A conceção de boa morte é ampla e sua compreensão propicia o fortalecimento sobre procedimentos relacionados à "morte digna".

\section{ABSTRACT}

Objectives: This research aimed to investigate the concept of dignified death for the Brazilian population.

Method: An exploratory, national survey, quantitative study was carried out. An inadvertent non-probabilistic sample of convenience was made up of 412 Brazilians who answered the "Scale of perception of worthy death", whose data were analyzed through descriptive and bivariate statistics using the statistical package SPSS (Statistical Package for Social Science) for Windows version 22 .

Results: It was possible to verify that decent death implies, in order of priority, a good relationship with the family $(M=$ $5.99, S D=0.73)$, maintenance of hope and pleasure $(M=5.88, S D=0.85)$, good relation with the professional health team $(M=5.46, S D=1.00)$, not to be a burden to the others $(M=5.38, S D=1.09)$, to have physical and cognitive control $(M=$ $4.69, S D=0.92)$ and control of the future $(M=4.57, S D=1.14)$.

Conclusions: The concept of good death is broad and its understanding provides for the strengthening of procedures related to "dignified death". 


\section{INTRODUÇÃO}

A morte e o morrer são vistos e refletidos de forma diferente ao longo da história, dependendo da cultura em que o indivíduo está inserido (Alonso, 2016; Ferreira, Souza e Lima, 2011). O conhecimento sobre morte trata acerca de assuntos que se estabelecem na vida, já que pesquisar sobre morte abrange a manifestação do homem interior. Na Idade Média, a morte era vista como um episódio impreterivelmente natural. Havia a aceitação da morte e, quando ela estava em iminência, as pessoas a assumiam e a vivenciavam em casa, junto com seus entes queridos, para se despedir (Ariès, 2003; Guandalini, 2010; Wanssa, 2012).

$\mathrm{Na}$ contemporaneidade, a morte mantém-se desagradável e misteriosa, causando receio, medo e temor. Sendo assim, existe uma inclinação do ser humano em evitá-la e negá-la, ou seja, é feito tudo para que a mesma não ocorra. As pessoas passaram a ver a morte como se a mesma não fosse inerente à vida, como se não fizesse parte das circunstâncias da vida. A morte, agora negada, tornou-se um tabu, onde as pessoas esquivam-se de comentar nos grupos e locais de socialização (Guandalini, 2010; Santana, Dutra, Carlos e Barros, 2017; Silva et al., 2016).

Nessa cultura, há dificuldade em deixar a vida seguir seu curso natural, especialmente entre os profissionais da saúde, que são formados e preparados para salvar e curar, e seu saber incorpora tecnologias que possibilitam o adiamento do processo de morte. Os aspetos relacionados ao fim da vida são associados à derrota, perda, fracasso e impotência e os investimentos em recursos biotecnológicos são alternativas para prolongar a vida do paciente, atendendo ao desejo de todos - paciente, família e equipe profissional (Combinato e Queiroz, 2006). Obstinação terapêutica realizada, muitas vezes, sabendo que o paciente tem prognóstico negativo, prolongando o sofrimento e a dor de todos os envolvidos, justificado pela não aceitação da morte. Torna-se, portanto, imprescindível a discussão sobre o impasse entre a atitude de intervir sobre a vida e morte, os métodos artificiais para prolongar a vida e a opção de deixar a doença seguir sua história natural com destaque para a eutanásia, a distanásia e a ortotanásia (Biondo, Silva e Secco, 2009; Felix et al., 2013; Silva et al., 2016).

A eutanásia foi inicialmente conceituada como uma ação de extrair a vida de uma pessoa, sem dor, sem angústia dispensável. Ultimamente, essa prática é apreendida para abreviar a vida, a fim de aliviar ou evitar a angústia dos pacientes. Aceito em alguns países como Holanda e Bélgica, é considerada crime no Brasil, pelo artigo 121 do Código Penal, embora seja juridicamente legal em alguns países (Castro et al., 2016; Felix et al., 2013).
A distanásia tem por definição a obstinação terapêutica marcada pela realização de tratamentos inúteis para salvar a qualquer custo a vida de um paciente. Trata-se da sustentação de tratamentos agressivos em doentes, que induz as pessoas ao artifício de morte branda, sofrida e apreensiva. Esta prática foi reforçada por muitos anos pelo Código de Ética Médica de 1931, que mostrava uma tendência a respaldá-la quando dizia: "um dos propósitos mais sublimes da medicina é sempre conservar e prolongar a vida" (Art. 16). No Código de 1988, entretanto, houve uma mudança de ênfase e, em seu Art. $2^{\circ}$ dizia que "o alvo da atenção do médico é a saúde da pessoa e deve agir com zelo em benefício da mesma". Apesar de tudo, a distanásia tem livre fluxo nos hospitais brasileiros, realizado com frequência de forma despercebida (Comin, Panka, Beltrame, Steffani e Bonamigo, 2017; Wanssa, 2012).

Já a ortotanásia é a morte digna. A origem da palavra, ortotanásia tem por definição morte correta - orto: certo; thanatos: morte. Relaciona-se ao processo de humanização da morte e ao alívio da dor, na qual a morte ocorre de forma desejável, de forma natural. Não se trata de negligência médica, mas da não realização de tratamentos invasivos, com enfrentamento da morte com dignidade, sem cair na distanásia (Felix et al., 2013). A ortotanásia respeita a vontade do paciente em processo de finitude e se opõe a intervenções que visualizam unicamente o prolongamento da morte no qual a dignidade e a autonomia do paciente é violada, e muitas vezes é operacionalizada por meio da prática de cuidados paliativos (Sanchez y Sanches e Seidl, 2013; Temel et al., 2010; Wanssa, 2012).

A partir dessa perspetiva, na tentativa de auxiliar os médicos nas decisões a serem tomadas, o Conselho Federal de Medicina, no ano de 2006, editou a Resolução $1.805 / 2006$ em que autoriza esses profissionais a limitarem ou suspenderem tratamentos e procedimentos que objetivavam prolongar a vida de pacientes com doenças graves e incuráveis em fase terminal e ainda em respeitar a vontade do paciente e de seus familiares ou representantes legais. Ato aceito e confirmado em 2010 pelo Ministério Público Federal que reconhece que a permissão para a interrupção do tratamento a pedido do doente em processo de finitude não fere a Constituição Federal. Desde então a ortotanásia é aceita pelo ordenamento jurídico. No campo da atuação dos profissionais da Medicina, suas atividades são regulamentadas pela Resolução n. 1.931/2009, o atual Código de Ética Médica, em que se pode entender que é dever do médico praticar a ortotanásia quando solicitada pelo paciente em processo de finitude (Conselho Federal de Medicina, 2006; Nunes, 2017).

Durante mais de três décadas, o interesse em melhorar o atendimento dos pacientes em processo de 
finitude deixou de ser a preocupação de alguns profissionais da saúde e passou a ser uma preocupação social generalizada. Apesar desta atenção, uma "boa morte" continua a ser mais uma esperança do que a prática médica padrão para todos os pacientes (Emanuel e Emanuel, 1998).

Diante dessa constatação, deve ser considerada a relevância de se promover a morte digna, para assegurar a qualidade do morrer. É preciso permitir aos pacientes escolherem o lugar onde desejam morrer e ajudar profissionais a não se culpabilizarem e não assumirem o fracasso por algo inerente ao processo de vida. Para tanto, faz-se necessário compreender a atual perceção da população sobre a morte digna, onde e como ela deve-se dar.

$\mathrm{Na}$ literatura, pode-se constatar algumas pesquisas sobre o tema. Wanssa (2012) realizou um estudo exploratório qualitativo com pacientes, familiares e profissionais de saúde a respeito de boa morte. Ele concluiu que, para uma boa morte, é primordial que exista uma comunicabilidade bem estruturada e clara que viabilize a tomada de decisão, integrada com pacientes e seus familiares, preparando esses para a morte, mediante a compreensão do que pode acontecer na trajetória que agora vai traçar, além de serem olhados como seres humanos até o fim. De forma complementar, o estudo de Menezes e Barbosa (2013) constatou que o lugar de propensão a uma boa morte é a casa do paciente e este deve ter autonomia para decidir se quer estar junto com seus entes queridos e amigos, em um clima acolhedor.

Nessa perspetiva, o estudo realizado por Silva et al. (2016) com enfermeiras da UTI de um hospital público da região do Vale do São Francisco verificou que os profissionais compreendem os princípios de eutanásia, distanásia e ortotanásia, e que percebem que este último colabora para uma morte digna, fazendo-se necessária a comunicação adequada entre paciente-família-equipe.

Steinhauser et al. (2000) retratam que, apesar de um aumento recente da atenção dada ao melhoramento dos cuidados de fim de vida, a nossa compreensão do que constitui uma boa morte é surpreendentemente faltante. Para mitigar esse problema, por meio de pesquisa com 65 participantes - incluindo médicos, enfermeiros, assistentes sociais, capelães, voluntários de hospício, pacientes e familiares recentemente detidos, os autores identificaram seis componentes principais de uma boa morte: gerenciamento de dor e sintomas, tomada de decisão clara, preparação para a morte, conclusão, contribuição para os outros e afirmação de toda a pessoa.
Ficou evidenciada, todavia, que são escassas as pesquisas sobre esse tema com foco sobre a perceção da população. A sua atualidade e a sua importância remetem ao interesse por estudá-lo, vez que visa buscar as explicações inerentes a esse olhar e por existirem poucas pesquisas sobre essa questão na realidade brasileira. Diante do exposto, o presente estudo objetivou compreender a perceção de morte digna para a população brasileira. A contribuição que se pretende para a ciência e para a sociedade é fomentar a humanização nos procedimentos adotados em pacientes terminais e, consequentemente, explorar a discussão sobre a desospitalização da morte.

\section{MÉTODO}

\section{Procedimentos}

Considerando-se os aspectos éticos referentes a pesquisas envolvendo seres humanos, o presente estudo foi submetido à Comissão de Ética em Pesquisa da Unifor, aprovado com parecer $N^{\circ} 1.310 .495$ de 05/09/2015. O instrumento foi disponibilizado na internet juntamente com o Termo de Consentimento Livre Esclarecido - TCLE, por meio de uma página específica e de domínio privado. A divulgação ocorreu durante quatro meses (agosto a dezembro de 2016) por meio de redes sociais on-line (Facebook) em divulgação de grupos de interesse em geral. Destaca-se ainda que foram respeitados os aspectos éticos exigidos pela Resolução no 466/12 do Conselho Nacional de Saúde.

\section{Amostra}

A população do Brasil é de 198,7 milhões de habitantes. Para uma população desse porte, com nível de confiança de $95 \%$ e erro amostral de 5\%, fez-se necessário uma amostra mínima de 385 participantes. Estes foram selecionados por meio de uma amostra não probabilística por conveniência (Cozby, 2003).

Ao final da coleta de dados, contou-se com uma amostra não probabilística acidental e por conveniência composta por 412 participantes. Teve como critério de inclusão ser brasileiro e maior de 18 anos, independente de outras características biodemográficas. Os participantes possuíam em média 29,48 $(D P=10,44)$ anos, variando entre 16 e 72 anos. A maioria dos participantes era mulher $(f=316 ; 76,90 \%)$, com ensino superior completo $(f=205 ; 49,90 \%)$, de diferentes cursos de todas as áreas do conhecimento, sem vínculo religioso $(f=179 ; 43,60 \%)$ e já tinha vivenciado a morte de alguém próximo $(f=356 ; 86,60 \%)$ (ver Tabela 1$)$. 
Tabela 1

Dados Sociodemográficos da Amostra $(N=412)$

\begin{tabular}{|c|c|c|c|c|}
\hline \multirow{3}{*}{ SEXO } & \multicolumn{2}{|c|}{ Homem } & \multicolumn{2}{|c|}{ Mulher } \\
\hline & \multicolumn{2}{|c|}{$f=95 ; 23,10 \%$} & \multicolumn{2}{|c|}{$f=316 ; 76,90 \%$} \\
\hline & $f=849 ; 41,70 \%$ & $f=142 ; 70 \%$ & $f=943 ; 45,30 \%$ & $f=124 ; 6,10 \%$ \\
\hline \multirow{2}{*}{ RELIGIÃO } & Sem vínculo & Católicos & Evangélico & Outras \\
\hline & $f=179 ; 43,60 \%$ & $f=132 ; 32,10 \%$ & $f=52 ; 12,70 \%$ & $f=28 ; 11,70 \%$ \\
\hline \multirow{2}{*}{ ESCOLARIDADE } & $\begin{array}{c}\text { EF incompleto } \\
\text { completo }\end{array}$ & EF completo & ES incompleto & ES completo \\
\hline & $f=06 ; 1,50 \%$ & $f=24 ; 5,80 \%$ & $f=176 ; 42,80 \%$ & $f=205 ; 49,90 \%$ \\
\hline \multirow{2}{*}{ TRABALHO } & Saúde & Humanas & $\begin{array}{c}\text { Sociais aplicadas } \\
\text { autônomo }\end{array}$ & Engenharias \\
\hline & $f=223 ; 54,30 \%$ & $f=66 ; 16,10 \%$ & $f=21 ; 5,10 \%$ & $f=25 ; 6,10 \%$ \\
\hline
\end{tabular}

Notas: $\mathrm{EF}=$ ensino fundamental; $\mathrm{ES}=$ ensino superior.

\section{Instrumentos}

Utilizou-se a "Versão reduzida da Escala de Percepção de Morte Digna", oriunda da "Escala de percepção de morte digna" (EPMD), originalmente de língua japonesa, criada por Miyashita, Sanjo, Morita, Hirai e Uchitomi (2007), e adaptada para o contexto brasileiro por Wanssa (2012). A versão utilizada é composta por 24 itens distribuídos em seis fatores: (1) manutenção da esperança e do prazer ( $\alpha=0,54 ; 7$ itens), que destaca os aspectos positivos da vida, o sentido de esperança atribuído e a sensação de que vale a pena viver; (2) boa relação com a equipe profissional de saúde $(\alpha=0,60 ; 10$ itens), que expressa a preocupação com as relações interpessoais, especificamente com as pessoas que são os principais cuidadores no contexto da enfermidade; (3) controle físico e cognitivo $(\alpha=0,57 ; 6$ itens), que mensura a importância de ser independente nas atividades (por exemplo, na alimentação e cuidados pessoais), o medo de demonstrar fraqueza e a capacidade de decisão; (4) não ser um fardo para os demais ( $\alpha=0,63 ; 7$ itens), que retrata o temor de ser dependente de equipamentos e/ou de outras pessoas; (5) boas relações com a família ( $\alpha=0,57$; 8 itens), que avalia a importância do apoio familiar, o conforto e desfrute do convívio com os parentes; e (6) controle do futuro $(\alpha=0,49 ; 4$ itens), que trata do conhecimento do tempo que resta de vida, de estar preparado para morrer e, principalmente, a disposição de despedida das pessoas queridas. Nela, os participantes precisavam indicar em que medida o teor expresso em cada item era necessário ou desnecessário para garantir uma morte digna, por meio de uma escala de respostas Likert que variava de 1 (totalmente desnecessário) a 7 (totalmente necessário) (ver Tabela 2).
Além disso, foi utilizado um questionário sociodemográfico para caracterizar a amostra por gênero, idade, escolaridade, região do país onde reside, religião e vivência de morte de alguém próximo.

\section{Tabela 2}

Distribuição dos Itens nos Fatores da Versão Reduzida da Escala de Perceção de Morte Digna

\begin{tabular}{ll} 
Fator & Abreviação do conteúdo do item \\
\hline I & Ter a sensação de que vale a pena viver \\
& Viver positivamente \\
& Viver como se estivesse em casa \\
& Viver em circunstâncias tranquilas \\
\hline II & Ser capaz de estar no lugar preferido \\
& Ter enfermeira com quem se sinta confortável \\
& Ter médico com quem conversar sobre morte \\
& Reconciliar-se com as pessoas \\
\hline III & Ser independente em atividades diárias \\
& Não demonstrar à família sua fraqueza \\
& Não mudar sua aparência \\
& Ser mentalmente capaz de tomar decisões \\
& Não ser um fardo para membros da família \\
& Não ser tratado como objeto ou criança \\
& Não ser dependente de equipamentos médicos \\
IV & Não trazer problemas para os demais \\
\hline & Contar com pessoas que possam ouvi-lo \\
& Desfrutar tempo suficiente com a família \\
& Estar calmo, relaxado \\
& Foram utilizados os tratamentos disponíveis \\
\hline & Saber adeus às pessoas queridas \\
& Controlar tempo de vida (eutanásia) \\
\hline & \\
\hline &
\end{tabular}




\section{Análise estatística}

As análises de dados foram realizadas com auxílio do pacote estatístico SPSS (Statistical Package for Social Science) for Windows versão 22, dividida em quatro etapas. Primeiro foi traçado o perfil da amostra, por meio de estatística descritiva (frequência, percentagem e medidas de tendência central e dispersão). Em seguida, foi realizada a análise descritiva dos fatores da escala. Para tanto, foi: 1) verificada a média da pontuação de cada fator (que variava entre 1 e 7 pontos); 2) realizada a interpretação da pontuação em quartil (1,00-1,75 Totalmente desnecessário; 1,76-3,50 - Desnecessário; 3,6-5,25 - Necessário; 5,26-7,00 - Totalmente necessário); 3) verificada a quantidade de participantes abaixo da média da amostra. Por fim, foram realizadas comparações das avaliações dos fatores e subfatores em função dos dados sociodemográficos: sexo, idade, escolaridade, curso e religião.

\section{RESULTADOS E DISCUSSÃO}

São apresentados nesta seção os resultados encontrados na pontuação de cada um dos seis fatores da "Versão reduzida da Escala de Perceção de Morte Digna", identificando o quanto cada um é necessário ou desnecessário para garantir uma morte digna (ver Tabela 3). Em seguida serão apresentados os resultados das comparações por dados sociodemográficos.

O Fator 1 - "Manutenção da esperança e do prazer" apresentou pontuação média de 5,88 ( $D P=0,85)$, variando entre 1,00 e 7,00 , representado no quartil "totalmente necessário". Observou-se ainda que 49 $(14,40 \%)$ participantes pontuaram abaixo da média. Nesse sentido, vale ressaltar que a maioria dos participantes da pesquisa destaca os aspetos positivos da vida, o sentido de esperança atribuído e a sensação de que vale a pena viver como aspetos relevantes para a morte digna. Realidade hoje reconhecida e que incita mudanças na forma de abordagem e tratamento aos pacientes terminais. A pesquisa de Morais, Nunes, Cavalcanti, Soares e Gouveia (2016) sobre a perceção de estudantes e profissionais de medicina sobre a morte digna evidenciou que a perceção na contemporaneidade sobre a morte na assistência hospitalar, exige mudanças radicais, exigindo aprendizados específicos que assumem maior espaço nos currículos na área da saúde, e incitando os hospitais a adquirir meios que permitam mais comodidade e cuidados a seus pacientes terminais.

O Fator 2 - "Boa relação com a equipe profissional de saúde" apresentou pontuação média de 5,46 ( $D P=$ $1,00)$, variando entre 1,25 e 7,00 , posicionado no quartil "totalmente necessário". Entre os participantes, 168 (40,60\%) foram apontados abaixo da média. Observa-se, portanto, que a maioria dos participantes da pesquisa acha importante para a morte digna a existência de boas relações interpessoais, principalmente com as pessoas que são os principais cuidadores no contexto da enfermidade. Nesse sentido, Santos e Bassitt (2011) mostram que a satisfação com o atendimento da equipe médica colabora na condição de final de vida. Para tanto, os familiares da pessoa que está enferma esperam dos profissionais atenção, informações honestas, conforto, respeito pelas emoções trazidas por esta situação e apoio espiritual, além de ouvi-las e responder sobre suas dúvidas.

A pontuação do Fator 3 - "Controle físico e cognitivo" foi de 4,69 ( $D P=0,92)$, com variações entre 1,00 e 6,75 , representado no quartil "necessário". Notase que $188(46,20 \%)$ sujeitos foram apontados abaixo da média. Pode-se compreender, portanto, que a maioria dos participantes da pesquisa acha importante para a morte digna ser independente nas atividades como alimentação e cuidados pessoais. Para Silva (2013) a aproximação da morte, normalmente é considerada pela idade avançada, em consideração de que é associado à velhice com perdas, tanto físico, quanto emocional e social.

O Fator 4 - "Não ser um fardo para os demais" apresentou pontuação média de 5,38 ( $D P=1,09)$, variando entre 1,00 e 7,00, posicionado no quartil "totalmente necessário". Observou-se ainda que 196 $(47,80 \%)$ sujeitos foram apontados abaixo da média. Ou seja, a maioria dos participantes tem temor de ser dependente de equipamentos e/ou de outras pessoas e não desejam isso no seu processo de morte. Corroborando com essa ideia, é abrangente a literatura em defesa do não uso de equipamentos para obstinação terapêutica de paciente em processo de finitude. Silva et al. (2016) defende que um cuidado em prol de minimizar a aflição da pessoa no procedimento da morte visa o descanso dos sintomas e desconfortos, poupando a condição de vida até o fim, de modo que a morte ocorra de forma natural. Igualmente, Silva (2013) mostra que pessoas de idade mais avançada agregam mais aversão à dependência do que à morte.

A pontuação do Fator 5 - "Boas relações com a família" apresentou-se com média de 5,99 ( $D P=0,73)$, variando entre 1,00 e 7,00 , representado no quartil "totalmente necessário". Observou-se ainda que 133 $(32,00 \%)$ sujeitos foram apontados abaixo da média. Para a maioria dos participantes é importante, portanto, o apoio familiar, o conforto e desfrute do convívio com os parentes no processo de morte. Nessa perspetiva, Santana et al. (2013) mostram que os familiares têm o poder de modificar a realidade dos pacientes terminais, inclusive sobre as decisões de distanásia e ortotanásia. Muitos aceitam e recebem o processo de morrer, enquanto alguns 
ordenam o investimento da equipe, adiando a angústia do paciente. Por outro lado, Santos e Bassitt (2011) revelam que no processo finitude é essencial o diálogo entre a equipe de profissionais e paciente/família, facilitando inclusive, a tomada de decisões.

O Fator 6 - "Controle do futuro" apresentou pontuação média de 4,57 $(D P=1,14)$, variando entre 1,25 e 7,00, representado no quartil "necessário". Entre os participantes, $203(48,90 \%)$ sujeitos foram apontados abaixo da média. Observa-se, portanto, que a maioria dos participantes considera relevante saber o tempo que resta de vida, de estar preparado para morrer e, principalmente, a disposição de despedida das pessoas queridas. Nessa perspetiva, Vane e Posso (2011) reforçam que, depois que aceitar que o paciente não tem cura, a terapia deve ser paliativa e não mais curativa, com o médico percebendo que ele deve avaliar a validade, os valores e opções que o paciente tem para investir no final da sua vida, levar o paciente a ser protagonista no procedimento de sua própria morte, que, se possível, estar em paz e próximo de seus amados.

A partir da comparação da pontuação desses seis fatores, destacam-se como aspetos mais importantes para a vivência de morte digna a existência de "Boas relações com a família" (morte sem isolamento), a "Manutenção da esperança e do prazer" (trazendo qualidade de morte) e a "Boa relação com a equipe profissional de saúde".

Por outro lado, detetou-se que, apesar de importantes, foram menos relevantes a preocupação em "Não ser um fardo para os demais" (dependência de outros ou de equipamentos), "Controle físico e cognitivo" e "Controle do futuro" (saber o tempo de vida e estar preparado para morrer).

Tabela 3

Pontuação dos Fatores da Versão Reduzida da Escala de Perceção de Morte Digna

\begin{tabular}{lccc}
\multicolumn{1}{c}{ Fator } & Ordem de importância & $\begin{array}{c}\text { Pontuação } \\
\text { Média }(\boldsymbol{D P})\end{array}$ & Quartil \\
\hline $\begin{array}{l}\text { 1. Manutenção da esperança e do prazer } \\
\text { 2. Boa relação com a equipe } \\
\text { profissional de saúde }\end{array}$ & 2 & $5,88(0,85)$ & Totalmente necessário \\
\hline $\begin{array}{l}\text { 3. Controle físico e cognitivo } \\
\text { 4. Não ser um fardo para os demais }\end{array}$ & 3 & $5,46(1,00)$ & Totalmente necessário \\
\hline $\begin{array}{l}\text { 5. Boas relações com a família } \\
\text { 6. Controle do futuro }\end{array}$ & 5 & $4,69(0,92)$ & Necessário \\
\hline
\end{tabular}

É possível verificar ainda que nas atribuições das pontuações de morte digna houve diferenças percebidas pelas comparações realizadas a partir de algumas variáveis sociodemográficas. Não apresentaram diferenças apenas as comparações por religião e nível de escolaridade. A seguir são apresentadas apenas as avaliações que se destacaram pela existência de diferenças estatisticamente significativas. Para tanto, destaca-se que todas as variáveis se apresentaram não normais a partir do teste de Shapiro-Wilk: fator $1(W=$ $0,87 ; p<0,01)$, fator $2(W=0,94 ; p<0,01)$, fator $3(W=$ $0,98 ; p<0,01)$, fator $4(W=0,96 ; p<0,01)$, fator $5(W=$ $0,82 ; p<0,01)$ e fator $6(W=0,99 ; p<0,01)$. Portanto, foram utilizados os testes não paramétricos MannWhitney e Kruskal-Wallis.

Foram apresentadas diferenças estatisticamente significativas nas comparações das respostas por gênero, sendo as maiores pontuações das mulheres: fator $2(U=$ $11605,50 ; p<0,05)$ [mulheres $(M=5,54 ; D P=0,99)$; homens $(M=5,19 ; D P=1,00)]$; fator $4(U=12862,50 ; p$
$<0,05)$ [mulheres $(M=5,44 ; D P=1,08)$; homens $(M=$ $5,20 ; D P=1,11)]$ e fator $5(U=12313,00 ; p<0,05)$ [mulheres $(M=6,05 ; D P=0,65)$; homens $(M=5,78 ; D P$ $=0,91)]$. Deste modo, para as mulheres é mais significativa a "Boa relação com a equipe profissional de saúde", "Não ser um fardo para os demais" e "Boa relação com a família".

Verificaram-se diferenças estatisticamente significativas nas comparações das pontuações por área de curso superior, no qual os do curso de saúde (Educação física, Enfermagem, Psicologia, Farmácia, Medicina e Odontologia) apresentaram maiores pontuações, seguido de engenharias: fator $2(14,34 ; p<0,05)$ [saúde $(M=5,60$; $D P=0,92)$; engenharias $(M=5,57 ; D P=0,94)$; humanas $(M=5,20 ; D P=1,20)$; ciências sociais aplicadas $(M=$ $4,76 ; D P=0,93)$ ] e fator $6(11,51 ; p<0,05)$ [saúde $(M=$ 4,77; $D P=1,05)$; engenharias $(M=4,43 ; D P=1,19)$; humanas $(M=4,39 ; D P=1,12)$; ciências sociais aplicadas $(M=3,92 ; D P=1,15)]$. Evidencia-se, portanto, que os profissionais de saúde consideram mais importante que os 
demais a "Boa relação com a equipe profissional de saúde" e o "Controle do futuro".

Nas comparações das pontuações do fator 2 e 6 entre os cursos de saúde, Enfermagem destacou-se com a maior pontuação no fator $2(M=5,92 ; D P=0,52)$ e Medicina no fator $6(M=5,01 ; D P=1,13)$. As pontuações mais baixas em ambos os fatores foram do curso de Odontologia: fator $2(M=4,88 ; D P=0,88)$ e fator $6(M=$ $3,63 ; D P=0,18)$.

\section{CONCLUSÃO}

Neste estudo foi pesquisada a perceção da população brasileira sobre morte digna, e possíveis distinções nas suas diversidades sociodemográficas: idade, sexo, curso superior (completo e incompleto), entre outros. Fundamentando-se em autores citados ao longo do artigo, verifica-se que a conceção de boa morte mudou ao longo da história. Sendo assim, boa morte seria relacionada à ortotanásia, morte correta - orto: certo; thanatos: morte. Pauta-se no processo de humanização da morte e alívio da dor, na qual a morte ocorre de forma desejável, de forma natural.

Foi possível verificar que a maioria dos participantes da pesquisa destaca os aspetos positivos da vida, o sentido de esperança atribuído e a sensação de que vale a pena viver como aspetos relevantes para a morte digna. Reforça-se que não está afirmando-se que estes fatores garantem que haja uma morte digna, ou que essas são condições determinantes; afirma-se sim, que os mesmos são considerados relevantes para a amostra de participantes.

Consegue-se ponderar como aspeto limitante deste estudo o fato de não ter sido possibilitado fazer uma pesquisa em longo prazo, de forma longitudinal, no sentido de entender fatores individuais e ambientais correlacionados à definição de boa morte. Propõe-se, que sejam realizadas outras pesquisas, investigando outras variáveis.

Finalizando, estima-se que os resultados deste estudo poderão colaborar para um melhor entendimento sobre a perceção de morte digna na população brasileira com o objetivo de colaborar para novos procedimentos de assistência no trabalho em saúde, tanto com familiares com pacientes em processo de finitude, bem como proporcionar o aprendizado e a pesquisa para a criação de novas conceções sobre o tema e para o fortalecimento sobre procedimentos relacionados à "morte digna".

Conflito de interesses | Conflict of interest: nenhum | none. Fontes de financiamento | Funding sources: nenhuma | none.

\section{REFERÊNCIAS}

Alonso, J. P. (2016). El derecho a una muerte digna en Argentina: La judicialización de la toma de decisiones médicas en el final de la vida. Revista de Saúde Coletiva, 26(2), 569-589. doi:10.1590/S0103-73312016000200012

Ariès, P. (2003). História da morte no Ocidente: Da idade média aos nossos dias. Rio de Janeiro: Ediouro.

Biondo, C. A., Silva, M. J. P. e Secco, L. M. D. (2009). Dysthanasia, euthanasia, orthotanasia: The perceptions of nurses working in intensive care units and care implications. Revista LatinoAmericana de Enfermagem, 17(5), 613-619. doi:10.1590/S010411692009000500003

Castro, M. P. R., Antunes, G. C., Marcon, L. M. P., Andrade, L. S., Rückl, S. e Andrade, V. L. A. (2016). Eutanásia e suicídio assistido em países ocidentais: Revisão sistemática. Revista Bioética, 24(2), 355-367. doi:10.1590/1983-80422016242136

Combinato, D. S. e Queiroz, M. S. (2006). Morte: Uma visão psicossocial. Estudos de Psicologia, 11(2), 209-216. doi:10.1590/S1413-294X2006000200010

Comin, L. T., Panka, M., Beltrame, V., Steffani, J. A. e Bonamigo, E. L. (2017). Percepção de pacientes oncológicos sobre terminalidade de vida. Revista Bioética, 25(2), 392-401. doi:10.1590/198380422017252199

Conselho Federal de Medicina. (2006). Resolução n. 1.805/2006 do Conselho Federal de Medicina. Brasília. Disponível em http://www.portalmedico.org.br/resolucoes/cfm/2006/1805_2006.htm

Cozby, P. C. (2003). Métodos de pesquisa em ciências do comportamento (1. ${ }^{\mathrm{a}}$ ed.). São Paulo, Brasil: Atlas S.A.

Emanuel, E. J. e Emanuel, L. L. (1998). The promise of a good death. The Lancet, 351(Special issue), SII21-SII29. doi:10.1016/S01406736(98)90329-4

Felix, Z. C., Costa, S. F. G., Alves, A. M. P. M., Andrade, C. G., Duarte, M. C. S. e Brito, F. M. (2013). Eutanásia, distanásia e ortotanásia: Revisão integrativa da literatura. Ciência \& Saúde Coletiva, 18(9), 2733-2746. doi:10.1590/S1413-81232013000900029

Ferreira, A. P. J., Souza, L. J. e Lima, A. A. F. (2011). O profissional de saúde frente à distanásia: Uma revisão integrativa. Revista Bioethikos, 5(4), 462-469. Recuperado em 11, fevereiro, 2017, em http://www.saocamilo-sp.br/pdf/bioethikos/89/A14.pdf

Guandalini, F. C. (2010). A transformação da relação do homem com a morte (Monografia de especialização, Universidade Católica do Paraná, Curitiba). Recuperado em 12, fevereiro, 2017, em http://www.symbolon.com.br/monografias/Felipe\%20Correa\%20 Guandalini\%20-

$\% 20$ AS $\% 20$ TRANSFORMACOES $\% 20$ DA $\% 20$ RELACAO $\% 20 D O$ \%20HOMEM\%20COM\%20A\%20MORTE.pdf

Menezes, R. A. e Barbosa, P. C. (2013). A construção da "boa morte" em diferentes etapas da vida: Reflexões em torno do ideário paliativista para adultos e crianças. Ciência \& Saúde Coletiva, 18(9), 2653-2662. doi:10.1590/S141381232013000900020

Miyashita, M., Sanjo, M., Morita, T., Hirai, K. e Uchitomi, Y. (2007). Good death in cancer care: A nationwide quantitative study. Annals of Oncology, 18(6), 1090-1097. doi:10.1093/annonc/mdm068

Morais, I. M., Nunes, R., Cavalcanti, T., Soares, A. K. S. e Gouveia, V. V. (2016). Percepção da "morte digna" por estudantes e médicos. Revista Bioética, 24(1), 108-117. doi:10.1590/198380422016241112

Nunes, L. (2017). Do ensino da bioética e as escolhas temáticas dos estudantes. Revista Bioética, 25(3), 512-526. doi:10.1590/198380422017253208

Sanchez y Sanches, K. M. e Seidl, E. M. F. (2013). Ortotanásia: Uma decisão frente à terminalidade. Interface - Comunicação, Saúde, Educação,17(44), 23-34. doi:10.1590/S141432832013000100003 
Santana, J. C. B., Dutra, B. S., Carlos, J. M. M. e Barros, J. K. A. (2017) Ortotanásia nas unidades de terapia intensiva: Percepção dos enfermeiros. Revista Bioética, 25(1), 158-167. doi:10.1590/198380422017251177

Santana, J. C. B., Santos, A. V., Silva, B. R., Oliveira, D. C. A., Caminha, E. M., Peres, F. S., ... Viana, M. B. O. (2013). Docentes de enfermagem e terminalidade em condições dignas. Revista Bioética, 21(2), 298-307. doi:10.1590/S1983-80422013000200013

Santos, M. F. G. e Bassitt, D. P. (2011). Terminalidade da vida em terapia intensiva: Posicionamento dos familiares sobre ortotanásia. Revista Brasileira de Terapia Intensiva, 23(4), 448454. doi:10.1590/S0103-507X2011000400009

Silva, C. C. (2013). A morte e a elaboração do luto na visão de alguns autores (Obtenção do título de especialista, Universidade Estadual do Vale do Acaraú, Brasil). Recuperado em $<$ https://psicologado.com/atuacao/tanatologia/a-morte-e-aelaboracao-do-luto-na-visao-de-alguns-autores $>$

Silva, R. S., Evangelista, C. L. S., Santos, R. D., Paixão, G. P. N., Marinho, C. L. A. e Lira, G. G. (2016). Percepção de enfermeiras intensivistas de hospital regional sobre distanásia, eutanásia e ortotanásia. Revista Bioética, 24(3), 579-589. doi:10.1590/198380422016243157
Steinhauser, K. E., Clipp, E. C., McNeilly, M., Christakis, N. A., McIntyre, L. M. e Tulsky, J. A. (2000). In search of a good death: Observations of patients, families, and providers. Annals of Internal Medicine,132(10), 825-832. doi: 10.7326/0003-4819-132-10200005160-00011

Temel, J. S., Greer, J. A., Muzikansky, A., Gallagher, E. R., Admane, S., Jackson, V. A., ... Lynch, T. J. (2010). Early palliative care for patients with metastatic non-small-cell lung cancer. Boston Medical and Surgical Journal, 363(8), 733-742. doi:10.1056/NEJMoa1000678

Vane, M. F. e Posso, I. P. (2011). Opinião dos médicos das Unidades de Terapia Intensiva do Complexo Hospital das Clínicas sobre a ortotanásia. Revista Dor, 12(1), 39-45. doi:10.1590/S180600132011000100009

Wanssa, M. C. D. (2012). Morte digna e lugar onde morrer: Percepção de pacientes oncológicos e de seus familiares. (Tese de Doutoramento, Faculdade de Medicina, Porto). Recuperado em 6, fevereiro, 2017, em $<$ https://sigarra.up.pt/ffup/pt/pub_geral.show_file?pi_gdoc_i $\mathrm{d}=519710>$ 